\title{
QUESTÕES SOBRE A DIMENSÃO INTERCULTURAL DA PERÍCIA PSICOLÓGICA NO SISTEMA JUDICIÁRIO FRANCÊS
}

\section{QUESTIONS ABOUT THE INTERCULTURAL DIMENSION OF THE EXPERT'S PSYCHOLOGICAL EXAMINATION IN THE FRENCH LEGAL SYSTEM}

Elaine Costa-Fernandez ${ }^{1}$

COSTA-FERNANDEZ, E. Questões sobre a dimensão intercultural da perícia psicológica no sistema judiciário francês. Rev. Bras. Cresc. Desenv. Hum., São Paulo, 12 (I), 2002.

\begin{abstract}
Resumo: A autora é perita judicial na comarca de Agen e professora de psicologia na Universidade de Toulouse le Mirail, onde ela dirige um seminário sobre os aspectos interculturais da perícia psicológica. Neste artigo ${ }^{2}$, ela expõe o caso de uma jovem vietnamita vítima de estupro por parte de um amigo de seus pais. A dimensão intercultüral da situação clínica estudada é dupla: ela se refere tanto ao confronto entre a cultura da pessoa examinada e a cultura da sociedade que vai julgar o crime quanto à distancia entre a abordagem psicológica e o mundo jurídico. Sua finalidade é fazer um elo entre lei e desejo, psiquismo e cultura. Questionar a dimensão intercultural desta intervenção permite, do ponto de vista teórico, a analise de mecanismos psíquicos específicos a toda situação de contato de culturas. Do ponto de vista metodológico, este trabalho inscreve-se no campo da psicologia clínica intercultural segundo a qual, as particularidades culturais do sujeito examinado fazem parte integrante da sua singularidade. Esta abordagem vai necessariamente alterar o diagnóstico de personalidade, além de introduzir uma revisão na interpretação de um teste projetivo como o Psicodiagnóstico de RORSCHACH. Desenvolve-se este enfoque segundo os três tempos de uma perícia psicológica: ao nível epistemológica, quando analisa as funções do psicólogo perito; no decorrer da instrução, quando o clínico encontra o sujeito, elabora suas hipóteses e redige o relatório; finalmente, durante o julgamento, na audiência, quando o perito apresenta suas conclusões diante dos jurados, participando assim aos debates. Do ponto de vista metodológico, a escolha de um único caso clínico visa evitar uma simplificação tipológica, respeitando a multiplicidade de situações.
\end{abstract}

Palavras-chave: abuso sexual; perícia psicológica; dimensão intercultural; Rorschach.

\section{O PSICÓLOGO INSCRITO COMO PERITO JUDICIAL}

\section{Desenvolvimento de uma especialidade}

Na França, todo e qualquer magistrado pode pedir ajuda a um técnico credenciado para responder questões específicas ligadas à sua disciplina, durante o inquérito ou durante a instrução do processo. Para isto, ele dispõe de listas de profissionais reconhecidos por suas competências técnicas e científicas.

O psicólogo clínico exercendo a função de perito judicial é um profissional independente,

1 Psicóloga clínica formada pela Universidade Federal do Rio de Janeiro (1981). Defendeu tese de doutorado na Universidade de Toulouse le Mirail em Psicologia Intercultural: Devenir sujet entre deux largues. L'aquisition du Français, largue seconde, chez des brésiliens vivant à Toulouse, sob a orientação de c. CLANET, (1991). Mestre de Conferência associada à Universidade de Toulouse le Mirail, professora convidada no instituto PIAGET, em Viseu, Portugal, pesquisadora do Centre d'Etudes e de Recherches em psychopathologie - CERPP, perita judicial junto à Corte de Apelo de Agen.

2 O estudo do caso que apresentaremos a seguir está inserido nos trabalhos de um seminário de mestrado, sob a nossa direção, na Universidade de Toulouse 11, sobre "As dificuldades metodológicas específicas da perícia psicológica em situação intercultural". 
inscrito como liberal, que aceita uma missão pontual que lhe é confiada pelo juiz de menores, juizados da vara de família ou encarregados da instrução do processo. Ele trabalha tanto para vara cível quanto para vara criminal. O recurso a este tipo de especialista é cada vez mais freqüente, o que provoca um aumento na procura de formações específicas e de temas de pesquisa.

A indicação de perícia psicológica tornouse sistemática cada vez que um menor é vitima de maus tratos, em casos de divórcios litigiosos, assim como nos processos de crime em que a responsabilidade do sujeito é colocada em questão. A intervenção do psicólogo clínico é complementar à do médico psiquiatra, mas ambas não se confundem. Apesar de ambas terem como origem comum a medicina legal, a perícia psicológica se afirma como uma especialidade autônoma e diferenciada.

\section{Especificidades da perícia psicológica rio meio judiciário}

A missão confiada ao psicólogo perito ultrapassa a determinação do diagnóstico e do prognóstico. Além de analisar elementos da personalidade como as características do funcionamento intelectual e a existência ou não de distúrbios da personalidade ou da conduta, é pedido ao profissional que avalie os efeitos de um determinado traumatismo na vida presente e futura da vítima, assim como uma eventual tendência à fabulação. Ele deve igualmente avaliar se fatos passados podem explicar, ao menos a nível de hipóteses, o aparecimento de tais desvios comportamentais ou de determinadas fragilidades.

Trata-se de uma intervenção imposta pela autoridade judicial, marcada pelos imperativos da ordem social, com o objetivo de assegurar o elo social. Ela se resume à uma visão pontual, precisa, feita em um momento determinado ${ }^{3}$. Neste contexto, a participação do sujeito independe da sua vontade, o que se opõe aos princípios éticos de todo encontro clínico.

\section{Confronto ou encontro intercultural?}

Para ultrapassar este paradoxo, o psicólogo clínico deve se basear nos aspectos interculturais da sua missão, ou seja, criar um espaço entre dois universos culturais distintos: psicologia e justiça. Ele deve oferecer ao sujeito um momento de escuta que lhe permita melhor integrar o processo jurídico, dar um sentido aos fatos, fazer emergir a culpabilidade ou reconhecer o seu lugar de vítima.
De forma geral, o papel exercido pelo psicólogo numa perícia judicial se caracteriza pela função de mediação entre a subjetividade do sujeito e a normalização imposta pela lei. Sua intervenção contribui para a apreensão dos valores culturais da justiça e do indivíduo.

Fazer surgir a interculturalidade, desta posição, consiste em interrogar o modo como estes dois discursos se completam, levando em conta suas especificidades e suas diferenças. Trata-se também de reconstruir o percurso pessoal e singular do sujeito examinado, levando-o a refletir sobre a distância que separa a sua história pessoal dos valores da sociedade que o acolhe.

Esta posição “entre dois”, a meio caminho entre realidade e fantasia, é bem mais delicada quando o sujeito examinado pertence a uma cultura estrangeira. Entretanto, nossa experiência, de mais de dez anos, como perita em psicologia confirma que, causa ou coincidência, a maioria dos indivíduos encontrados são originários de uma cultura estrangeira à sociedade francesa.

\section{O CASO KIM}

Kim é uma jovem de origem vietnamita, nascida na França, que declara ter sido vítima de abusos sexuais por parte de um amigo de seus pais. Nós a encontramos a pedido do Juiz de instrução. Nossa missão consistia em avaliar os efeitos do traumatismo bem como discernir uma tendência eventual a fantasiar, afabular.

\section{Elementos da situação pessoal e familiar}

Encontramos Kim na casa de seus pais. $\mathrm{O}$ apartamento era limpo, bem arrumado, decorado com objetos religiosos vindos do Vietnã, país de origem da família. Nele havia inúmeras plantas verdes, um aquário, budas e fotos de antepassados nas paredes. Todo o ambiente nos transportou à Asia. Ficamos surpreendidos pelas diferenças culturais entre o interior do apartamento e o conjunto habitacional no qual ele estava localizado, donde nós nos interrogarmos sobre os efeitos psicológicos de tal ruptura intercultural.

Kim é uma adolescente de 14 anos e 7 meses, com uma aparência tímida e submissa que declara não ter jamais encontrado o seu pai biológico. Ela foi reconhecida pelo atual companheiro de sua mãe, residente na França há 25 anos. Ele foi dono de restaurante, mas agora está desempregado. O casal não é casado, estão juntos há 11 anos. Um filho nasceu dessa união. Ele cria as

3 Nonnalmente o psicólogo dispõe de 3 meses para realisar sua tarefa. O número de sessões varia entre I e 4 sessões, dependendo do professional e da complexidade da situação. 
três crianças de sua companheira como se fosse o pai. Ele próprio tem dois filhos de outro casamento, que também vivem com eles.

Na entrevista clínica, o padrasto de Kim mostrou-se desestabilizado pelas revelações. Reagiu afetivamente, na medida em que se sente pessoalmente atingido no papel de chefe de família. Segundo ele, a honra e a moral são prioritárias, face às eventuais dificuldades da vítima. Ele admite ter sido rígido e não ter diálogo com a jovem, apesar de amá-la como se fosse sua própria filha.

A mãe de Kim, de 46 anos de idade, trabalhou no restaurante de seu companheiro mas, neste momento, encontra-se igualmente desempregada. Ela só fala vietnamita em casa. Diz mal saber ler e escrever francês. Ela se preocupa com a transmissão cultural e gostaria que as crianças conservassem os costumes e as tradições do país de origem.

No entanto, todos os filhos nasceram na França. Eles nunca tiveram dificuldades na escola. Eles terão a nacionalidade francesa com a maioridade. O padrasto de Kim confirma ser duro com eles, tê-los esbofeteado, batido. Ele reconhece adotar o método tradicional para educar suas crianças. Isto pode justificar o medo da jovem em contar os fatos aos pais. Contudo, ele se declara satisfeito de descobrir “a verdade” e diz confiar na legislação francesa.

\section{Análise do discurso}

Ouvida individualmente, Kim reconstitui globalmente os fatos dos quais se diz vítima, sem entrar nos detalhes. Kim não reivindica a punição do acusado. Nenhuma revolta transparece no seu discurso. Segundo sua mãe, Kim era uma jovem muito alegre, mas o trauma a tornou triste. Mudanças significativas de comportamento e de caráter foram constatadas depois da revelação.

Ela fala francês com fluência, de modo simples, direto, mas considera-se "mais vietnamita que francesa”. Diz também gostar do inglês. A representação de si própria é bem elaborada, mas seu conteúdo é depressivo, e sua aparência é fechada.

No que diz respeito aos planos para o futuro, Kim se diz disposta a respeitar a vontade dos pais. Contudo, acaba por revelar o sonho de partir para longe, de viajar, de viver no Canadá ou nos Estados Unidos. Gostaria também de ser escritora, mas tem medo das dificuldades materiais que iria enfrentar. Ao mesmo tempo romântico e angustiado, o discurso de Kim refere-se regularmente a uma cisão interna, a uma dificuldade de se assumir, a uma ambivalência. Seu discurso contém elementos inquietantes indicando um risco de despersonalização.
“... Ter duas culturas é bom... eu sou uma pessoa sem cara... me escondo atrás de uma personalidade que não sou eu mesma... Creio que o que sofri me fez mais madura..."

\section{Análise de suas interpretações no teste de Rorschach}

O protocolo de Kim, elaborado a partir das manchas de tinta do teste de Rorschach, é rico, variado, nenhuma recusa é constatada. Ela leva em consideração os elementos sensoriais das manchas, particularmente as cores. À medida que o teste evolui, constatamos um maior investimento afetivo, com mais distanciamento do material. Pouco a pouco verifica-se o predomínio do imaginário sobre o real:

P l.l “... Eu não sei o que é... podem ser duas pessoas que seguram outra pessoa e ali, são as mãos da pessoa que não quer ir embora. Mas eles o levam... como nos filmes... ele... ele não quer ir mas os outros... levam ele pró inferno... eu não gosto do preto... é triste...” Pl. 2 “... Eu não sei... eu não sei de nada... podem ser duas pessoas de mãos dadas, mas que morrem juntas, já que tem o vermelho. Eles se suicidam os dois..."

A maneira de apreender a prancha é, em sua maior parte, global, com a percepção de certos detalhes vindo justificar a interpretação. O funcionamento intelectual parece normal. Suas interpretações são bastante criativas, originais.

Pl. 4 “... Elas são o tempo todo assim? Tenho a impressão que é o mal que vem... que vai nos invadir como a tristeza que cresce dentro da cabeça... avança, avança... depois, por ser preto, vai entrar no coração... gosto desta, é bonita..."

Pl. 5 “... Ah... diríamos que é uma borboleta que se transforma em um morcego... porque o morcego vive no escuro... é por isso que ele é preto... e, mais, ele é feio. Ao contrário da borboleta... quando ela se torna morcego, é o contrário dela mesma. Pode ser que ela seja preta no exteriorl e em cores por dentro. Ela tem garras para se proteger...".

A pertinência das interpretações ilustra a qualidade da percepção da realidade. Há uma tentativa de encenação com um número importante de movimentos, o que supõe a existência de um 
mundo interior e de capacidades de projetar seu imaginário sobre a imagem.

Pl. 6 “... Ah, é divertido... este é o seu corpo...tem o mal que tenta invadí-lo por baixo e ele f ca cada vez menor..” Pl. 7 “... pode-se dizer duas pessoas que querem se juntar, mas elas não podem. Elas tentam, mas não podem... Elas tentam sempre... isto representa a coragern”. Pl. 9 “... isto agora, são pessoas, e isto, isto representa o bem, a alegria, e aquilo, é a tristeza. Isto quer dizer que numa pessoa sempre tem o mal e o bem. Não se pode nunca ser o mal perfeito...".

Sua principal dificuldade consiste em dar uma forma aos perceptos. Os engramas são maioritariamente vagos, seus limites são imprecisos. Ela está sujeita à invasão de fantasmas e a trasbordamentos afetivos. Ela fornece um número importante de interpretações abstratas, sem determinantes formais definidos.

Pl. 10. "Então, isso são duas pessoas... Elas estão mortas... Elas vão ao paraíso e há dois anjos... não, de fato há dois demônios... elas vão ao paraíso... elas vão ao inferno. Há demônios que os acompanham e eles estão sempre unidos... Eles estão ligados... sobre uma nuvem... Eles vão para a liberdade, mas eles estão cada vez mais submetidos. Eles podem ir para uma luz, muito brilhante, mas, de fato, está muito escuro... Não é muito brilhante...”.

\section{Apresentação dos resultados do estudo}

O relatório feito pelo perito, e entregue ao Juiz, deve ser lido pelos advogados ao sujeito. Trata-se de uma apresentação do exame clínico, dos resultados dos testes de personalidade e de nível intelectual. Ele deve levar em conta tanto a linguagem jurídica quanto os conceitos psicológicos ao responder às perguntas formuladas na missão.

Ao nível psicopatológico, poderíamos fazer as seguintes hipóteses diagnósticas:

- O diagnóstico de debilidade pode ser afastado. Ela apresenta um funcionamento intelectual variado, próximo à média previsivel para a sua idade.

- Ela consegue investir os processos mentais, principalmente no nível da síntese. A análise fina dos detalhes assim como a atenção dirigida ao material são mínimas.

- Ela tem dificuldades de controle e de domínio afetivo. Uma tendência impulsiva clara transparece em sua produção.
- A invasão de afetos disfóricos de tendência depressiva é massiva.

- Boa adaptação à realidade. Percepção estruturada do real, o que nos permite afastar a existência de perturbações graves de personalidade.

- Problemas conflituosos testemunham uma estruturação neurótica da personalidade. A alteridade está confirmada. Por outro lado, falta autonomia e diferenciação aos personagens.

No entanto, certos elementos do protocolo sugerem o diagnóstico de organização de falsoself. Isto porque sua produção está carregada de abstrações e de referências simbólicas, pelas quais ela manifesta uma grande predileção.

Nota-se igualmente a presença de um certo tipo de impressões cinéticas. Segundo CHABERT (1983), o falso-ego se apresenta como querendo ir "ao fundo das coisas a fim de encontrar sua essência”. Este tipo de funcionamento psíquico pouco procura quais poderiam ser as configurações das manchas, os perceptos possíveis. No lugar de uma "borboleta”, ela nos fala de uma "impressão de liberdade". Ela nos faz parte de suas impressões e expõe seu estado de espírito, com várias referências autobiográficas. Podemos observar um importante investimento do subjetivo. Trata-se de um impressionismo aonde o sentido importa mais do que o percebido.

Em compensação, se nos referirmos ao contexto cultural de Kim, suas respostas parecem bem menos patológicas. A tendência introvertida, impressionista, de suas interpretações corresponde a modelos culturalmente valorizados. A problemática da despersonalização, da cisão identitária transparece em certos relatos:

Pl. 8. “... Lá, há dois animais... eles tentam atingir o cume, mas eles não conseguem. O cume, diríamos que ele cresce todo o tempo, nunca se chega... tenta-se sempre atingí-lo, mas não se pode... E lá, este de cores vivas, eles estão contentes, felizes e mais eles sobem, mais eles estão tristes... mais isto se torna escuro...”.

\section{DISCUSSÃO}

Como perita, participei dos debates no Supremo tribunal de Justiça em matéria criminal "Cour d'Assises". Os fatos relatados por Kim foram julgados por um juri popular ao término da instrução. $\mathrm{O}$ acusado, que terminou por assumir a autoria do crime de estupro, foi condenado a 5 anos de prisão. 
A dimensão intercultural da intervenção, neste caso, foi dupla. Por um lado, tentei subjetivar os efeitos do traumatismo, analisar suas repercussões no percurso pessoal e familiar da vítima. Em seguida, trouxe elementos sobre o meio cultural da família, principalmente no que diz respeito às relações entre as gerações e à importância dada à palavra da criança no Viet-Nam. Minha intervenção foi uma tentativa de explicação do silêncio e da submissão de Kim à autoridade do pai. Finalmente, o maior interesse deste caso clínico consiste na presença de elementos interculturais no discurso e no protocolo de Rorschach. A posição “entre dois” de Kim, neste sentido, é análoga à da perita e ao encontro clínico.

\section{CONCLUSÃO}

Da entrevista clínica, destacamos a desadaptação social e sofrimento psíquico de Kim. A testagem realizada a partir de testes projetivos nos permite constatar uma organização neurótica da personalidade, se bem que elementos próximos ao funcionamento limite sejam igualmente percebidos. Além disso, afetos depressivos e uma importante falta de confiança em si alertam o profissional para o risco de passagem a aios depressivos.

Fazemos a hipótese de que Kim não consegue alcançar uma identidade estável por não ser suficientemente flexível para "navegar" entre referências tão afastadas.

Fazemos a hipótese de que as produções de Kim são a tradução de uma cisão cultural, de uma dificuldade de integração da noção de interdito em relação com o dever de uma transmissão cultural imposta.

Em situação de perícia psicológica, o clínico intervém a partir de uma indicação social, mas o encontro clínico pode fazer emergir a questão própria ao sujeito. A dimensão terapêutica de sua intervenção reside em o sujeito do processo judicial levar em consideração a elaboração de sua relação com a Lei, do reconhecimento de seu lugar como vítima, ou bem de sua responsabilidade na passagem ao ato -, e não em uma atitude reparadora do clínico.

O psicólogo perito não se limita à compilação descritiva dos sintomas, mesmo se a missão que lhe é confiada lhe pede um diagnóstico, um prognóstico. Ele vai funcionar como uma interface, mediador entre sistemas de referências, entre universos de significações contraditórias, se não opostas. Seu relatório deverá ser compreendido e defendido pelos advogados, em uma linguagem jurídica, a partir de conceitos psicológicos.

A situação intercultural se caracteriza por sua ambigüidade. Simultaneamente possibilidade de ligação, de encontro entre universos culturais diferentes, e ruptura, confrontação, entre representações pessoais e valores coletivos institucionalizados. A dimensão intercultural de toda situação clínica supõe um esforço de contextualização dos mecanismos em jogo. Ela implica em uma explicitação dos processos psíquicos específicos à situação estudada.

Através de exemplos como este, a abordagem da psicologia intercultural nos traz instrumentos e uma reflexão metodológica nova. Além de questionar os modelos existentes, ela permite a apreensão de zonas de encontros entre universos culturais tão distantes como o dos psicólogos, com noções como imaginário, fantasmas, inconsciente, seu código de classificação de doenças, o segredo profissional, e o do judiciário, com a determinação de regras sociais e os limites das transgressões, segundo os Códigos Civil e Penal.

Enfatizar o campo intercultural é trazer a atenção dos atores sociais para os processos psicológicos e sociais que subentendem as tentativas do sujeito para manter uma identidade de si tanto pessoal quanto social; é tentar discernir suas tentativas para transformar e manter esta identidade em função das situações novas às quais ele está confrontado. 


\begin{abstract}
The author is a legal expert in the district court of Agen and a psychology professor in the Univensity of Toulouse le Mirail, where she coordinates a seminar on the intercultural a spects of the expert's psychological examination. In this article, she presents the case of a young Vietnamese who was raped by a friend of her parents. The intercultural dimension of the studied clinical situation is twofold: it refens to the conflict between the culture of the examined penson and the culture of the society that will judge the crime, and also to the distance between the psychological approach and the legal world. Its purpose is to build a linkbetween law and desire, psychicism and culture. he questioning of the intercultural dimension of this intervention allows, from the theoretical point of view, the analysis of psychic mechanisms that are specific to every situation of contact between cultures. From the methodological point of view, this work is inserted in the field of intercultural clinical psychology, according to which the cultural particularities of the examined subject are an integral part of his/her uniqueness. This approach will certainly alter the personality diagnosis. In addition, it will introduce a review ofthe interpretation of a projective test like RORSCHACH's Psychodiagnosis. It is developed according to the three moments of an expert's psychological examination: in the epistemological levei, when she analyses the functions of the expert psychologist; during the proceedings, when the clinician meets the subject, elaborates his hypotheses and writes the report; finally, during the hearing, when the expert presents his conclusions in front of the members of the jury, participating in the debates. From the methodological point of view, the choice of asingle clinical case has the purpose of avoiding a typological simplification, respecting the multiplicity of situations.
\end{abstract}

Key-words: sexual abuse; expert's psychological examination; intercultural dimension; Rorschach.

\section{REFERÊNCIA BIBLIOGRÁFICA}

CHABERT, C. Le Rorschach en clinique adulte. Paris: Dunod, 1983.
KISS, A. Psychiatrie communautaire au Viêt-Nam: ville, campagne et réinsertion sociale. In: HILY, M. A.; LEFEBVRE, C. (Dir.). Identités collective et altérité. Paris: L’Harmattan, 1999.

Recebido em 15/01/2002

Modificado em 02/02/2002

Aprovado em 10/03/2002

4 Indicações bibliográficas: CAMILLERI, C.; VINSONNEAU, G. Psychologie et culture, concepts et méthodes. Paris: Armand Colin, 1996; CLANET, C. L'interculturel, introduction. Toulouse: PUM, 1990; COSTA-FERNANDEZ, E. L'utilisation du TAT en expertise psychologique, une étude de cas. In: ..Villerbu, L.; Viaux, J. L. Expertise psychologigue, psychopathologie et méthodologie. Paris: L’Hannattan, 1999. p. 325-333; COUCHARD, F. La psychologie clinique interculturelle. Paris: Dunod, 1999. (Coll. Les Topos); DUFLOT-FAVORI, C. Le psychologuc en justice. Paris: PUF, 1988. (Coll. Le psychologue); GUERRAOUI, Z.; BERTRAND, T. Psychologic interculturelle. Paris: Armand Colin, 2000. (Coll. Synthèse); GUERRAOUI, Z.; TROADEC, B. Psychologie interculturelle. Paris: Armand Colin, 2000. (Coll. Synthèse); IDRIS, I. L’innovation et le temps de l'intégration de l'altérité; chronique de l'autre. Journal des Psycholoque, février: 55-58, 2001; LACOMBE, L. Expertise psychologique en contexte pluriethnique. Journal des Psycholoques, octobre: 4749, 2000; RAYMOND, S. Les expertises en sciences humaines. Toulouse: Privat, 1989; VIAUX, J. L. Victimes: actes et silences. Rouen: Publications de l’Université de Rouen, 1995. 